\title{
MRI contrast agents: Classification and application (Review)
}

\author{
YU-DONG XIAO, RAMCHANDRA PAUDEL, JUN LIU, CONG MA, ZI-SHU ZHANG and SHUN-KE ZHOU
}

Department of Radiology, The Second Xiangya Hospital of Central South University, Changsha, Hunan 410011, P.R. China

Received August 20, 2015; Accepted July 13, 2016

DOI: $10.3892 /$ ijmm.2016.2744

\begin{abstract}
Magnetic resonance imaging (MRI) contrast agents are categorised according to the following specific features: chemical composition including the presence or absence of metal atoms, route of administration, magnetic properties, effect on the magnetic resonance image, biodistribution and imaging applications. The majority of these agents are either paramagnetic ion complexes or superparamagnetic magnetite particles and contain lanthanide elements such as gadolinium $\left(\mathrm{Gd}^{3+}\right)$ or transition metal manganese $\left(\mathrm{Mn}^{2+}\right)$. These elements shorten the $\mathrm{T} 1$ or $\mathrm{T} 2$ relaxation time, thereby causing increased signal intensity on T1-weighted images or reduced signal intensity on T2-weighted images. Most paramagnetic contrast agents are positive agents. These agents shorten the T1, so the enhanced parts appear bright on T1-weighted images. Dysprosium, superparamagnetic agents and ferromagnetic agents are negative contrast agents. The enhanced parts appear darker on T2-weighted images. MRI contrast agents incorporating chelating agents reduces storage in the human body, enhances excretion and reduces toxicity. MRI contrast agents may be administered orally or intravenously. According to biodistribution and applications, MRI contrast agents may
\end{abstract}

Correspondence to: Professor Shun-Ke Zhou, Department of Radiology, The Second Xiangya Hospital of Central South University, 139 Middle Renmin Road, Changsha, Hunan 410011, P.R. China

E-mail:shk@medmail.com.cn

Abbreviations: MRI, magnetic resonance imaging; Gd, gadolinium; $\mathrm{Mn}$, manganese; Dy, dysprosium; SPIO, superparamagnetic iron oxide; USPIO, ultrasmall superparamagnetic iron oxide; SIPP, superparamagnetic iron platinum particle; Mn-DPDP, manganese dipyridoxyl diphosphate or mangafodipir trisodium; MEMRI, manganese-enhanced MRI; Gd-DTPA, gadolinium (III) diethylenetriamine pentaacetate; Gd-DOTA, gadoterate dotarem; Gd-EOB-DTPA, gadolinium ethoxybenzyl diethylenetriamine pentaacetate or gadoxetate; $\mathrm{Cr}$, chromium; Gd-DTPA-BMA, gadolinium 3-diethylenetriamine pentaacetate-bis(methylamide); Gd-HP-DO3A, gadoteridol; Gd-BOPTA, gadobenate dimeglumine; OCA, oral contrast agent; GI, gastrointestinal; CT, computed tomography; ECF, extracellular fluid; BPCA, blood pool contrast agent; HSA, human serum albumin; RES, reticuloendothelial system

Key words: magnetic resonance imaging, contrast agents, classification, application be categorised into three types: extracellular fluid, blood pool and target/organ-specific agents. A number of contrast agents have been developed to selectively distinguish liver pathologies. Some agents are also capable of targeting other organs, inflammation as well as specific tumors.

\section{Contents}

1. Introduction

2. Magnetic properties

3. Chemical composition and the presence or absence of metal atoms

4. Route of administration

5. Effect on the magnetic resonance image

6. Biodistribution and applications

7. Future prospects and conclusions

\section{Introduction}

Magnetic resonance imaging (MRI) contrast agents are widely used to increase the contrast difference between normal and abnormal tissues. Shortly after the introduction of clinical MRI, the first contrast-enhanced human MRI study was reported in 1981 using ferric chloride as the contrast agent in the gastrointestinal (GI) tract (1). In 1984, Carr et al first proved the use of a gadolinium compound as a diagnostic intravascular MRI contrast agent (2). Almost half of the MRI studies performed nowadays are contrast-enhanced studies, and this is a growing trend (3). Newer contrast agents are constantly being discovered and investigated. The safety of contrast agents for clinical use is under strict scrutiny. This review therefore, aims to classify the MRI contrast agents discovered to date into relevant groups and to also discuss their applications, structures, mechanisms of action, pharmacokinetics and pharmacodynamics.

MRI contrast agents may be categorised according to the following features (4): magnetic properties, chemical composition, the presence or absence of metal atoms, route of administration, effect on the magnetic resonance image, biodistribution and application.

\section{Magnetic properties}

The majority of MRI contrast agents are either paramagnetic gadolinium ion complexes or superparamagnetic (iron oxide) 
magnetite particles. The paramagnetic contrast agents are usually made from dysprosium $\left(\mathrm{Dy}^{3+}\right)$, the lanthanide metal gadolinium $\left(\mathrm{Gd}^{3+}\right)$, or the transition metal manganese $\left(\mathrm{Mn}^{2+}\right)$ and possess water soluble properties. The most commonly selected metal atom used in MRI contrast agents is the lanthanide ion gadolinium (III) as it possesses a high magnetic moment and it is the most stable ion with unpaired electrons. Due to the presence of unpaired electrons, these contrast agents possess paramagnetic properties; gadolinium has seven, dysprosium has four and manganese has five unpaired electrons. Contrast agents containing gadolinium shorten the T1 (or longitudinal) and T2 (or transverse) relaxation time of neighbouring water protons (Fig. 1). These effects increase the signal intensity of T1-weighted images, and reduce the signal intensity of T2-weighted images $(5,6)$. T1 shortening occurs at lower gadolinium concentrations, whereas $\mathrm{T} 2$ shortening occurs at higher gadolinium concentrations, which is of limited clinical use due to the increased risk of toxicity. Therefore, in conventional clinical practice $\mathrm{T} 1$ is evaluated after the administration of extracellular agents (7). Contrast agents containing transition metal ions, such as high spin manganese (II) and superparamagnetic iron oxide such as iron (III) oxides, affect the T2 relaxation strongly $(8,9)$.

Gadolinium-based contrast agents: paramagnetic. Gadolinium (III)-based contrast agents are categorised into three groups: extracellular fluid (ECF) agents, blood pool contrast agents (BPCAs) and organ-specific agents.

Manganese-based contrast agents: paramagnetic. Manganese, in the form of manganese chelates or manganese-based nanoparticles, is used as a contrast agent. Manganese chelates, including manganese dipyridoxyl diphosphate (Mn-DPDP), markedly enhance the T1 signal intensity, and has been used to detect hepatic lesions. In the human body, the chelate dissociates into manganese and DPDP. Manganese is taken up by the liver cells and excreted into the bile, whereas the DPDP component is excreted by the kidneys (10). Research on Mn-based nanoparticles is not as detailed in comparison with other well-studied nanoparticles based on iron oxide (11).

Manganese-enhanced MRI (MEMRI) uses manganese ions $\left(\mathrm{Mn}^{2+}\right)$ and this contrast agent has applications in animal experiments (12). $\mathrm{Mn}^{2+}$ enters cells through calcium $\left(\mathrm{Ca}^{2+}\right)$ channels and thus, this group of contrast agents may be used for functional brain imaging (13). A previous MRI study has suggested that $\mathrm{Mn}^{2+}$ carbon nanostructure complexes of graphene oxide nanoplatelets and graphene oxide nanoribbons are highly effective MRI contrast agents (14).

Iron oxide contrast agents: superparamagnetic. There are two types of iron oxide contrast agents: superparamagnetic iron oxide (SPIO) and ultrasmall superparamagnetic iron oxide (USPIO). Superparamagnetic contrast agents consist of suspended colloids of iron oxide nanoparticles. When applied during imaging, they reduce the intensity of the T2 signals in the tissues which absorb the contrast agent. SPIO and USPIO have achieved successful outcomes in the diagnosis of liver tumors in some cases (15). Two decades ago, SPIO was the first nanoparticulate MRI contrast agent to be introduced as a liver contrast agent, and it is still used for clinical imaging (16). SPIOs and USPIOs such as Feridex I.V., Resovist, Sinerem and
Clariscan have been approved for use in the past. However, these agents are currently unavailable apart from the oral iron oxide contrast agent, Lumirem/GastroMARK.

The nano-sized dimensions and the particle shapes of this group of contrast materials allow for different biodistribution and applications that are not observed with other contrast agents. At present, nanoparticulate iron oxide is a popular and unique nanoparticulate agent used in clinical practice. However, owing to the sophisticated modern technology of molecular and cellular imaging, which makes disease-specific biomarkers visible at microscopic and molecular levels, other nanoparticles have also obtained greater attention as potential MRI contrast agents. Due to the enormous improvement in nanotechnology, novel nanoparticulate MRI contrast agents have been developed with further improved contrast abilities as well as other functions (16).

Iron platinum contrast agents: superparamagnetic. Compared with iron oxide nanoparticles, superparamagnetic iron platinum particles (SIPPs) are thought to possess significantly improved T2 relaxation properties. SIPPs have been encapsulated with phospholipids to create multifunctional SIPP stealth immunomicelles in order to specifically target human prostate cancer cells (17). These contrast agents are still under investigation and have not yet been studied in humans, to the best of our knowledge. This study revealed that multifunctional SIPP micelles have been synthesized and conjugated to a monoclonal antibody against prostate-specific membrane antigen. In addition, the complex specifically targeted human prostate cancer cells in vitro, suggesting that SIPPs may have the potential to be tumor-specific in the future (17).

\section{Chemical composition and the presence or absence of metal atoms}

MRI contrast agents may be divided into two classifications as mentioned previously. The first group is comprised of paramagnetic compounds, which include lanthanides such as gadolinium. The second group is comprised of transition elements such as manganese and iron.

In order to reduce the toxicity of metal ions, the concept of chelation has been introduced. To prepare contrast agents based on metallic ions, the technique of chelated complex formation is widely used. The acute and the chronic toxic side-effects induced by the metal ion as well as the chelating agent are markedly reduced due to complexation (18).

As mentioned previously, gadolinium is used as a gadolinium (III) ion. Gadolinium (III) is weakly bound to serum proteins and may be displaced by ligands. Lanthanide salts generally hydrolyse into hydroxides, which are taken up by the reticuloendothelial system (RES) and accumulate in the body, particularly in the liver, spleen and bone, thereby causing potential toxicity. Lanthanide ions are excreted into both urine and faeces, unlike manganese ions which are almost exclusively excreted by GI elimination, via the biliary route. To overcome the aforementioned problems, these elements are administered in chelated forms.

Gadolinium-based chelation complexes. Fig. 2 illustrates the gadolinium-based chelation complexes used in clinical practice. There are three types of gadolinium (III)-based chelates (18). 


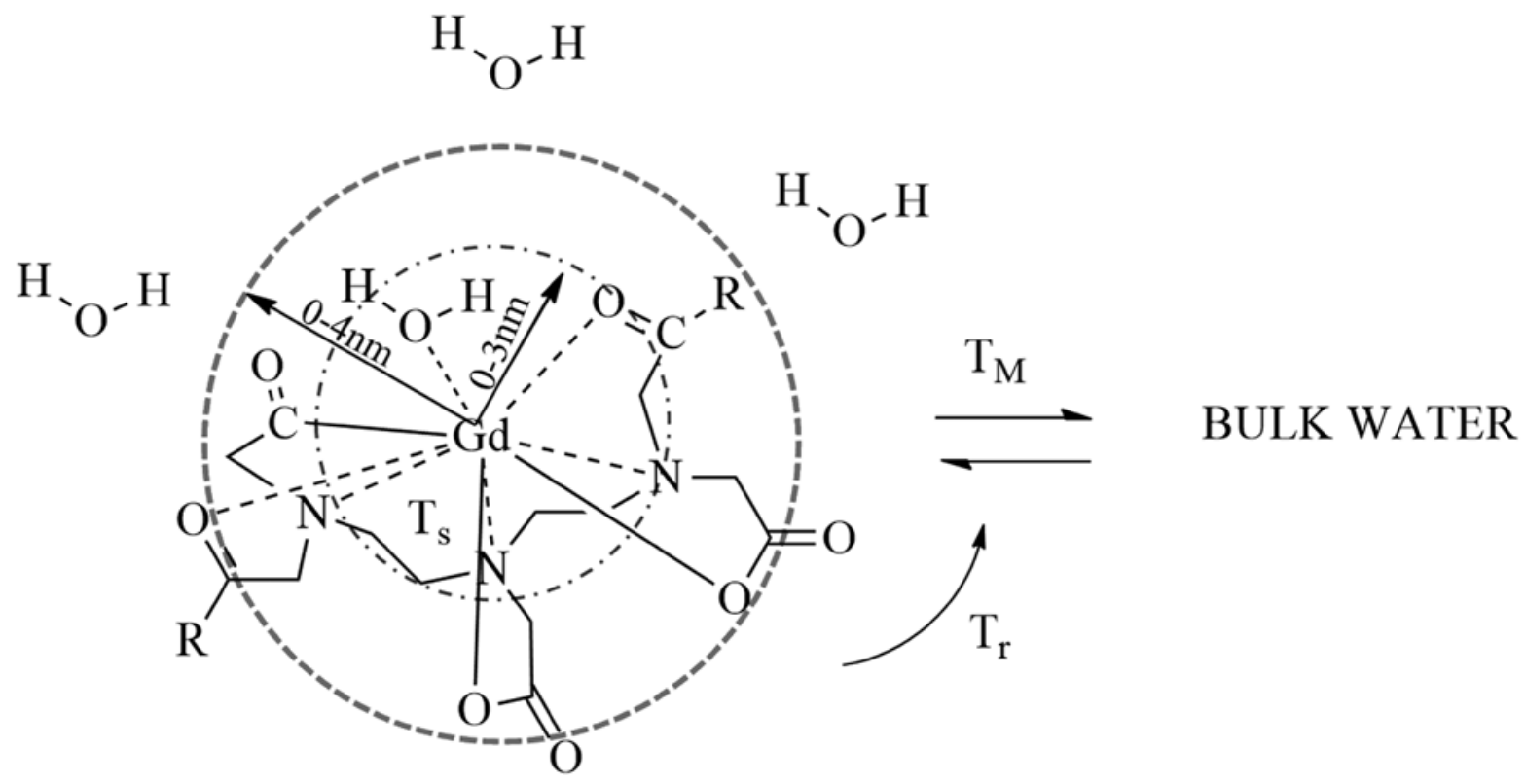

Figure 1. Interactions between gadolinium complexes and water resulting in relaxation of water protons.

Ionic and hydrophilic complexes. Ionic and hydrophilic complexes include gadolinium (III) diethylenetriamine pentaacetate (Gd-DTPA, also known as gadopentate dimeglumine), Gd(III) 1,4,7,10-tetrazacyclododecane NN'N"N"'-tetra-acetate (Gd-DOTA, gadoterate) (19) and Gd(III) polyaspartate.

Nonionic and hydrophilic complexes. Nonionic hydrophilic chelates of gadolinium (III) include Gd3-diethylenetriamine pentaacetate-bis(methylamide) (Gd-DTPA-BMA, also known as gadodiamide) and a macrocyclic chelate analog of Gd-DOTA, where an acetic acid function is replaced by a 2-propanol radical (Gd-HP-DO3A, also known as gadoteridol) (20).

Ionic and lipophilic complexes. The other group of gadolinium complexes includes the Gd benzyl-oxy-methyl derivative of diethyltriamine pentaacetate dimethylglucamine salt (Gd-BOPTA, also known as gadobenate dimeglumine) and $\mathrm{Gd}$ ethoxybenzyl diethylentriamine pentaacetate (Gd-EOB-DTPA, also known as gadoxetate) (18).

\section{Route of administration}

MRI contrast agents may be administered intravenously or orally. The route of administration is dependent on the subject of interest. A list of contrast agents is presented in Table I.

Intravenous contrast agents. Intravenous MRI contrast agents are comprised of chelates of paramagnetic ions, both ionic and nonionic. The particulates are isolated in the liver, spleen and lymph nodes. The intravascular agents are confined to the blood pool and to specific tumors.

Ionic intravenous contrast agents. The first intravenous contrast agents to be used were the chelates of paramagnetic ions $\mathrm{Cr}$ and $\mathrm{Gd}$ in combination with ethylenediaminetetraacetic acid (EDTA). However, EDTA was relatively unstable, and was found to cause toxic effects in an animal study (21). Gd-DTPA has been successfully used due to its stability and reliability, and it is the first intravenous MRI contrast agent to be approved for use in humans (Magnevist; Berlex Laboratories). Gd has a large magnetic moment, exceeded only by dysprosium (III) and holmium (III). Even at low concentrations, it possesses strong paramagnetic properties and low toxicity. The paramagnetic properties are due to the fact that it has seven unpaired electrons, as stated previously. Following intravenous administration, it is distributed in the intravascular and extracellular fluid spaces, and then rapidly excreted into urine (22).

Nonionic intravenous contrast agents. Nonionic contrast agents have been developed in parallel with iodinated contrast materials. Some side effects are due to the fact that ionic chelates are hyperosmolar. In contrast to ionic agents, nonionic agents are relatively hypoosmolar. Gadodiamide (Omniscan; Winthrop Pharmaceuticals) is a nonionic complex, which has only two-fifths of the osmolality of Gd-DTPA. Owing to a median lethal dose of $34 \mathrm{mmol} / \mathrm{kg}$, gadodiamide has a safety ratio 2-3 times that of Gd-DOTA, and 3-4 times that of Gd-DTPA. The administration of gadodiamide does not cause abnormalities in serum bilirubin levels. However, one study conducted in 73 individuals demonstrated that elevated serum iron levels are a potential concern with an incidence of $8.2 \%$, and a similar efficacy to that of Gd-DTPA (23). Gadoteridol (ProHance; Squibb) is the third kind of intravenous contrast agent sold on the market. It is a nonionic contrast agent with osmolarity similar to that of gadodiamide (24).

MRI oral contrast agents (OCAs). The oral administration of contrast agents is appropriate for GI tract scans. Naturally prepared fruit juices such as Medlar fruit juice, blueberry juice and green tea, have been studied as MRI contrast agents for several years. Artificial OCAs are based on the heavy metal ions such as gadolinium, manganese (III), manganese (II), 


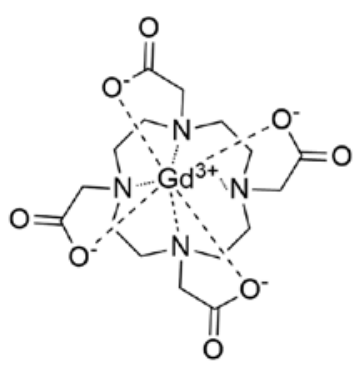

Gd-DOTA

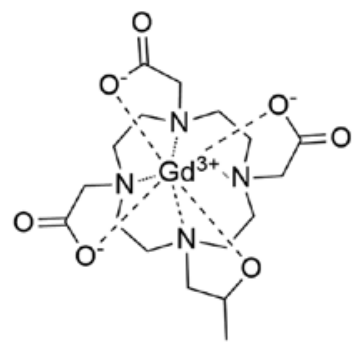

Gd-HP-DO3A

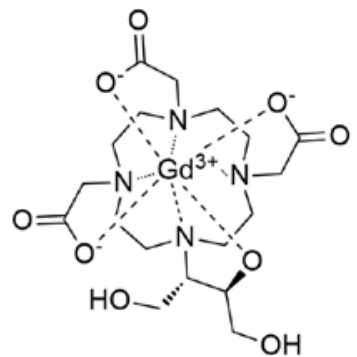

Gd-BT-DO3A

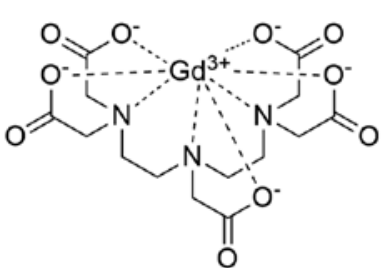

Gd-DTPA

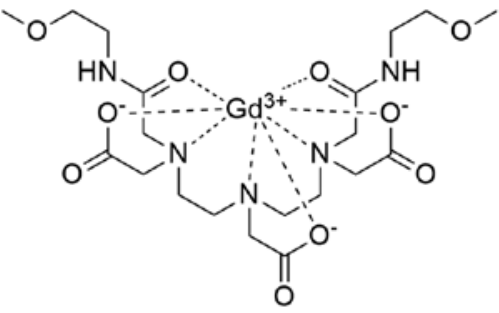

Gd-DTPA-BMEA

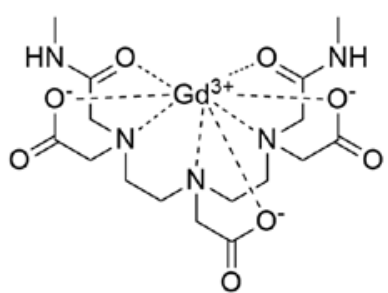

Gd-DTPA-BMA

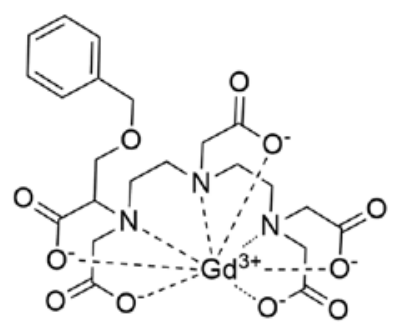

Gd-BORTA

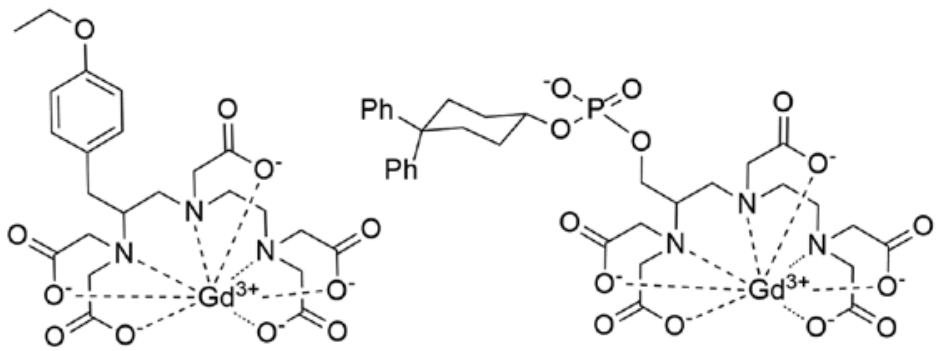

Ms-325

Figure 2. Clinically used contrast agents (CAs) based on Gd(III) complexes.

copper (II) and iron (III). Air and clay are used to reduce the T2 signal intensity $(25,26)$. Gadolinium-based agents, SPIO, manganese-containing agents and barium sulfate suspensions have been studied as oral MRI contrast agents $(27,28)$. The oral administration of MRI contrast agents containing manganese is a novel, noninvasive method for imaging (27). Barium sulfate suspensions are useful as negative oral MRI contrast agents (28). Divalent manganese ions are paramagnetic and greatly reduce the $\mathrm{T} 1$ relaxation time, thereby increasing $\mathrm{T} 1$ signal intensity. Manganese enters excitable cells such as neurons and myocardiocytes through various calcium channels. Thus, manganese acts as an indicator of calcium channel activity (27). However, the intravascular route of administration of MRI contrast agents is more useful and is the more commonly used route for MRI scans.

\section{Effect on the image}

Paramagnetic contrast agents, apart from dysprosium-based compounds, are positive agents and they exert similar effects on T1 imaging and T2 imaging. However, as the T1 of tissues is much higher than the $\mathrm{T} 2$, the predominant effect at low does is that of T1 shortening (29). The tissues absorbing such agents become bright on T1-weighted images.

Negative contrast agents reduce T2 signals by shortening the T2 relaxation time. Superparamagnetic and ferromagnetic agents belong to this group. However, reducing the particle size of ferromagnetic particles size results in the permanent loss of magnetic properties, and a change to become superparamagnetic particles (30). These compounds may also become T1 agents, depending on particle size and coating.

\section{Biodistribution and applications}

Extracellular fluid (ECF) agents. ECF agents (so called intravenous contrast agents) are distributed within the extracellular space. These agents have been used for the longest period of time in liver imaging, and they remain the most commonly used and well-documented. ECF agents are comprised of gadolinium chelated to an organic compound such as DTPA (31-33). A list of the ECF agents is presented in Table II. 
Table I. Agents administered orally.

\begin{tabular}{llll}
\hline Short name & \multicolumn{1}{c}{ Generic name } & \multicolumn{1}{c}{ Trade name } & Enhancement \\
\hline Gd-DTPA $^{\mathrm{a}}$ & Gadopentate dimeglumine & Magnevist Enteral & Positive \\
- $^{\mathrm{a}}$ & Ferric amonium citrate & Ferriseltz & Positive \\
$-^{\mathrm{a}}$ & Manganese chloride & LumenHance & Positive \\
-a $^{\text {aMP }}$ & Gadolinium-loaded zeolite & Gadolite & Positive \\
AMI-121 $^{\mathrm{b}}$ & Ferristene (MPIO) & Abdoscan & Negative \\
PFOB $^{\mathrm{b}}$ & Ferumoxsil (MPIO) & Lumirem/GastroMARK & Negative \\
\hline
\end{tabular}

${ }^{\mathrm{a} A g e n t s ~ a v a i l a b l e ~ f o r ~ c l i n i c a l ~ a p p l i c a t i o n ; ~}{ }^{\mathrm{b}}$ agents withdrawn from market. MPIO, micron size iron oxide particles. This table has been modified from http://www.magnetic-resonance.org/ch/13-01.html.

Table II. ECF space agents.

\begin{tabular}{llll}
\hline Short name & \multicolumn{1}{c}{ Generic name } & Trade name & Enhancement and physiochemical effects \\
\hline Gd-DTPA $^{\mathrm{a}}$ & Gadopentate dimeglumine & Magnevist & Positive-ionic-linear \\
Gd-DOTA $^{\mathrm{a}}$ & Gadoterate meglumine & Dotarem, Artirem & Positive-ionic-macrocyclic \\
Gd-DTPA-BMA $^{\mathrm{a}}$ & Gadodiamide injection & Omniscan & Positive-nonionic-linear \\
Gd-HP-DO3A $^{\mathrm{a}}$ & Gadoteridol injection & ProHance & Positive-nonionic-macrocylic \\
Gd-DTPA-BMEA $^{\text {a }}$ & Gadoversetamide & OptiMARK & Positive-nonionic-linear \\
Gd-DO3A-butrol $^{\text {a }}$ & Gadobutrol & Gadovist & Positive-nonionic-macrocyclic \\
Gd-BOPTA $^{\text {a }}$ & Gadobenate dimeglumine & MultiHance & Positive-ionic-linear
\end{tabular}

a, Agents available for clinical application. ECF, extracellular fluid; Gd-DTPA, gadolinium (III) diethylene triamine pentaacetate; Gd-DOTA, gadoterate dotarem; Gd-DTPA-BMA, gadolinium 3-diethylenetriamine pentaacetate-bis(methylamide). This table has been modified from http://www.magnetic-resonance.org/ch/13-01.html.

The pharmacokinetics of gadolinium chelates mimic that of iodinated contrast agents for computed tomography (CT). The contrast agents circulate and then freely distribute in the extracellular space. ECF agents are mainly eliminated by renal excretion. Gadolinium enters the liver through the hepatic artery and portal vein, and is freely redistributed into the interstitial space. In contrast to iodine molecules which are imaged by $\mathrm{CT}$, the effect of gadolinium is assessed by MRI rather than the molecule itself. Gadolinium exhibits an amplification effect as a number of adjacent water protons are relaxed by a single gadolinium atom. As a result, MRI is more sensitive to the effects of gadolinium than CT is to the effects of iodine $(29,33)$.

BPCAs. BPCAs, also known as intravascular contrast agents, remain in the intravascular space much longer and are excreted more slowly than their ECF counterparts, thus providing a longer time window for the imaging of blood vessels. These agents are currently under investigation for use in angiography, which may be performed in the equilibrium phase (34). A list of BPCAs is presented in Table III.

The BPCAs may be classified into the following three categories based on their mechanism of action: i) systems based on the noncovalent binding of low-molecular Gd to human serum albumin (HSA) to prevent immediate leakage into the interstitial space $(35,36)$; ii) systems incorporating polymers or liposomes based on an increase in the size of the contrast agent, which slows down leakage through endothelial pores $(37,38)$; and iii) systems based on nanoparticles, involving a change in the route of elimination (39-41). These agents may be classified broadly into three categories: USPIO particles, agents that reversibly bind to plasma proteins, and macromolecules $(42,43)$.

Targeted and organ-specific contrast agents. The primary aim of MRI contrast agent development is to identify agents which are capable of targeting specific tissues. A list of such compounds is presented in Table IV. It is important to consider the following three parameters in order to optimize the development of targeted and organ-specific contrast agents: i) improvement of the enhancing effect as high and ultrahigh fields call for a different contrast agent compared with low and medium high fields; ii) selective distribution in the body as it is necessary for these contrast agents to accumulate (organ-or pathology-specific tracers) at the required site in order to reach high local concentrations and iii) improvement of tolerance: although tolerance of existing compounds is already very good, this means, the compound has to be inert chemically and biologically, and also has to be completely eliminated from the body. 
Table III. BPCAs.

\begin{tabular}{|c|c|c|c|}
\hline Short name & Generic name & Trade name & Enhancement \\
\hline $\mathrm{NC}-100150^{\mathrm{b}}$ & PEG-feron (USPIO) & Clariscan & Positive \\
\hline SH U $555 \mathrm{C}^{\mathrm{b}}$ & Ferucarbotran (USPIO) & Supravist & Positive \\
\hline MS-325 & Gadofosveset & AngioMARK, Vasovist, Ablavar & Positive \\
\hline Gadomer- $17^{\mathrm{b}}$ & - & - & Positive \\
\hline Gabofluorine- $\mathrm{M}^{\mathrm{b}}$ & - & - & Positive \\
\hline $\mathrm{P} 792^{\mathrm{b}}$ & Gadomelitol & Vistarem & Positive \\
\hline AMI- $227^{\mathrm{c}}$ & Ferumoxtran-10 (USPIO) & Sinerem/Combidex & Positive or negative \\
\hline Gd-BOPTA $^{\mathrm{a}}$ & Gadobenate dimeglumine & MultiHance & Positive \\
\hline
\end{tabular}

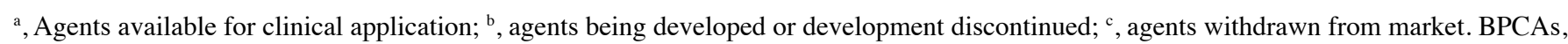
blood pool contrast agents; USPIO, ultrasmall superparamagnetic iron oxide. This table has been modified from http://www.magnetic-resonance.org/ch/13-01.html.

Table IV. Targeted/organ-specific agents.

\begin{tabular}{|c|c|c|c|}
\hline Short name & Generic name & Trade name & Enhancement and physiochemical effects \\
\hline Mn-DPDP ${ }^{c}$ & Mangafodipir trisodium & Treslascan & Positive/liver \\
\hline Gd-EOB-DTPA ${ }^{\mathrm{a}}$ & Gadoxetate & Primovist, Eovist & Positive-ionic-linear/liver \\
\hline Gd-BOPTA $^{\mathrm{a}}$ & Gadobenate dimeglumine & MultiHance & Positive-ionic-linear/liver \\
\hline $\mathrm{AMI}-25^{\mathrm{a}}$ & Ferumoxides (SPIO) & Endorem, Feridex & Negative/liver \\
\hline SH U $555 \mathrm{~A}^{\mathrm{c}}$ & Ferucarbotran (SPIO) & Resovist, Cliavist & Negative/liver \\
\hline AMI- $227^{c}$ & Ferumoxtran-10 (USPIO) & Sinerem, Combidex & Positive or negative/lymph nodes \\
\hline Gadofluorine- $\mathbf{M}^{\mathrm{b}}$ & - & - & Positive/(lymph nodes, CNS) \\
\hline Mn-DPDP & Mangafodipir trisodium & - & Positive/myocardium \\
\hline Dy-DTPA-BMA ${ }^{b}$ & Sprodiamide injection & - & Negative/myocardial and brain perfusion \\
\hline Gd-DTPA-mesoporphyrin ${ }^{\mathrm{b}}$ & Gadophrin & - & Positive/myocardium, necrosis \\
\hline
\end{tabular}

Iron oxides and liposomes have attracted particular interest as potential organ-specific agents. Iron oxide particles are imported into the cells of the RES through phagocytosis, which provides selective access to the liver, spleen, lymph nodes, and bone marrow. These agents can either be positive (T1) or negative (T2/T2*) enhancers, depending on particle size, composition, concentration and saturation magnetization of the material as well as the equipment hardware and pulse sequences used. The biodistribution of iron oxides is determined by size, shape, charge, hydrophilicity, chemical composition and surface coating (44). The majority of compounds are polydisperse and polycrystalline. However, actively targeted iron oxides, which tend to contain smaller superparamagnetic labels, are monodisperse and monocrystalline. For intravenous use, iron oxide particles should be $<50 \mathrm{~nm}$ in order to avoid entrapment in the lungs.

Another group of particulate contrast agents are liposomes. Paramagnetic ions may either be encapsulated in the aqueous compartment of the liposomes or be linked to their lipid bilayers. More sophisticated liposome compounds have been developed including phospholipid spin-labeled and amphipathic chelate complexes.

The primary organ selected for developing passive targeting compounds (vascular, hepatobiliary, and reticuloendothelial) is the liver. In addition to vascular structures, both hepatocytes and the RES may be targeted. By dynamic examinations, vascular structures as well as highly vascularized lesions are commonly highlighted with the conventional low molecular weight contrast agents. Both Gd-EOB-DTPA and Gd-BOPTA are positive gadolinium-based agents with lipophilic side groups. Gd-EOB-DTPA is a liver-specific agent whereas Gd-BOPTA is a multipurpose contrast agent, well suited for liver imaging (45). Mn-DPDP is a positive multipurpose agent, which taken up by hepatocytes (46). Contrast enhancement appears to be due to the limited release of the manganese ion and this effect is long lasting and may be achieved with doses as low as $10 \mathrm{mmol} / \mathrm{kg}$ body weight. 
Further applications. Some contrast agents may also be capable of targeting other organs such as the spleen, pancreas, bone marrow, lymph nodes, adrenals, muscles and particularly the heart as well as inflammation and specific tumors. However, they are not yet ready for use in clinical practice.

\section{Future prospects and conclusions}

The first MRI contrast agent to be used was ferric chloride in 1981. Over the past 3 decades, many contrast agents have been developed for use in clinical practice and some of them were withdrawn as result of safety concerns. The MRI contrast agents discovered to date may be classified into various groups according to a number of criteria: chemical composition, the presence of metal atoms, route of administration, magnetic properties, effect on the image, biodistribution and further applications. As a result there are variations in the clinical implications, mechanisms of action, safety, pharmacokinetics and pharmacodynamics of these contrast agents. Currently, newer and safer MRI agents capable of targeting organs, sites of inflammation and specific tumors are under investigation in order to develop contrast agents with higher disease specificity.

\section{References}

1. Young IR, Clarke GJ, Bailes DR, Pennock JM, Doyle FH and Bydder GM: Enhancement of relaxation rate with paramagnetic contrast agents in NMR imaging. J Comput Tomogr 5: 543-547, 1981.

2. Carr DH, Brown J, Bydder GM, Weinmann HJ, Speck U, Thomas DJ and Young IR: Intravenous chelated gadolinium as a contrast agent in NMR imaging of cerebral tumours. Lancet 1: 484-486, 1984.

3. Tang JB, Sheng YQ, Hu HJ and Shen YQ: Macromolecular MRI contrast agents: structures, properties and applications. Prog Polym Sci 38: 462-502, 2013.

4. Geraldes CFGC and Laurent S: Classification and basic properties of contrast agents for magnetic resonance imaging. Contrast Media Mol Imaging 4: 1-23, 2009.

5. Mitchell DG: Liver I: Currently available gadolinium chelates. Magn Reson Imaging Clin N Am 4: 37-51, 1996.

6. Wood ML and Hardy PA: Proton relaxation enhancement. J Magn Reson Imaging 3: 149-156, 1993.

7. Gandhi SN, Brown MA, Wong JG, Aguirre DA and Sirlin CB: MR contrast agents for liver imaging: what, when, how. Radiographics 26: 1621-1636, 2006.

8. Shokrollahi H: Contrast agents for MRI. Mater Sci Eng C 33 4485-4497, 2013.

9. Yurt A and Kazanci N: Investigation of magnetic properties of various complexes prepared as contrast agents for MRI. J Mol Struct 892: 392-397, 2008.

10. Harisinghani MG, Jhaveri KS, Weissleder R, Schima W, Saini S, Hahn PF and Mueller PR: MRI contrast agents for evaluating focal hepatic lesions. Clin Radiol 56: 714-725, 2001.

11. Zhen ZP and Xie J: Development of manganese-based nanoparticles as contrast probes for magnetic resonance imaging. Theranostics 2: 45-54, 2012.

12. Silva AC,Lee JH, Aoki L and Koretsky AR: Manganese-enhanced magnetic resonance imaging (MEMRI): methodological and practical considerations. NMR Biomed 17: 532-543, 2004.

13. Lin YJ and Koretsky AP: Manganese ion enhances T-1-weighted MRI during brain activation: An approach to direct imaging of brain function. Magn Reson Med 38: 378-388, 1997.

14. Paratala BS, Jacobson BD, Kanakia S, Francis LD and Sitharaman B: Physicochemical characterization, and relaxometry studies of micro-graphite oxide, graphene nanoplatelets, and nanoribbons.. PloS One 7: e38185, 2012.

15. Nakamura H, Ito N, Kotake F, Mizokami Y and Matsuoka T: Tumor-detecting capacity and clinical usefulness of SPIO-MRI in patients with hepatocellular carcinoma. J Gastroenterol 35: $849-855,2000$.
16. Na HB, Song IC and Hyeon T: Inorganic nanoparticles for MRI contrast agents. Adv Mater 21: 2133-2148, 2009.

17. Taylor RM, Huber DL, Monson TC, Ali AMS, Bisoffi M and Sillerud LO: Multifunctional iron platinum stealth immunomicelles: targeted detection of human prostate cancer cells using both fluorescence and magnetic resonance imaging. J Nanopart Res 13: 4717-4729, 2011.

18. Thunus L and Lejeune R: Overview of transition metal and lanthanide complexes as diagnostic tools. Coordin Chem Rev 184: 125-155, 1999.

19. Sijens PE, van den Bent MJ, Nowak PJ, van Dijk P and Oudkerk M: $1 \mathrm{H}$ chemical shift imaging reveals loss of brain tumor choline signal after administration of Gd-contrast. Magn Reson Med 37: 222-225, 1997.

20. Chang CA: Magnetic resonance imaging contrast agents. Design and physicochemical properties of gadodiamide. Invest Radiol 28 (Suppl 1): S21-S27, 1993.

21. Runge VM, Clanton JA, Herzer WA, Gibbs SJ, Price AC, Partain CL and James AE Jr: Intravascular contrast agents suitable for magnetic resonance imaging. Radiology 153: 171-176, 1984.

22. Runge VM, Schoerner W, Niendorf HP, Laniado M, Koehler D, Claussen C, Felix R and James AE Jr: Initial clinical evaluation of gadolinium DTPA for contrast-enhanced magnetic resonance imaging. Magn Reson Imaging 3: 27-35, 1985.

23. Kaplan GD, Aisen AM and Aravapalli SR: Preliminary clinical trial of gadodiamide injection: a new nonionic gadolinium contrast agent for MR imaging. J Magn Reson Imaging 1: 57-62, 1991.

24. Runge VM, Dean B, Lee C, Carolan F and Heard G: Phase III clinical evaluation of Gd-HP-DO3A in head and spine disease. J Magn Reson Imaging 1: 47-56, 1991.

25. Cordova-Fraga T, Sosa M, Hernandez-Gonzalez MA, Reyes-Aguilera JA, Solorio S, Ramirez C, Bautista-Flores E, Reynaga G, Avila-Rodriguez M and De la Roca-Chiapas JM: Medlar (Achras sapota L.) as oral contrast agent for MRI of the gastrointestinal tract. Appl Magn Reson 42: 161-167, 2012.

26. Mayo-Smith WW: Computed body tomography with MRI correlation. AJR Am J Roentgenol 173: 266, 1999.

27. Jacobs KE, Behera D, Rosenberg J, Gold G, Moseley M, Yeomans D and Biswal S: Oral manganese as an MRI contrast agent for the detection of nociceptive activity. NMR Biomed 25: 563-569, 2012.

28. Li KC, Tart RP, Fitzsimmons JR, Storm BL, Mao J and Rolfes RJ: Barium sulfate suspension as a negative oral MRI contrast agent: in vitro and human optimization studies. Magn Reson Imaging 9: 141-150, 1991.

29. Verloh N, Utpatel K, Haimerl M, Zeman F, Fellner C, Fichtner-Feigl S, Teufel A, Stroszczynski C, Evert $M$ and Wiggermann P: Liver fibrosis and Gd-EOB-DTPA-enhanced MRI: A histopathologic correlation. Sci Rep 5: 15408, 2015.

30. Weissleder R, Bogdanov A and Papisov M: Drug targeting in magnetic resonance imaging. Magn Reson Q 8: 55-63, 1992.

31. Ahmad MW, Xu W, Kim SJ, Baeck JS, Chang Y, Bae JE, Chae KS, Park JA, Kim TJ and Lee GH: Potential dual imaging nanoparticle: Gd2O3 nanoparticle. Sci Rep 5: 8549, 2015.

32. Edelman RR, Siegel JB, Singer A, Dupuis K and Longmaid HE: Dynamic MR imaging of the liver with Gd-DTPA: initial clinical results. AJR Am J Roentgenol 153: 1213-1219, 1989.

33. Balci NC and Semelka RC: Contrast agents for MR imaging of the liver. Radiol Clin North Am 43: 887-898, 2005.

34. Nolte-Ernsting C, Adam G, Bücker A, Berges S, Bjørnerud A and Günther RW: Abdominal MR angiography performed using blood pool contrast agents: comparison of a new superparamagnetic iron oxide nanoparticle and a linear gadolinium polymer. AJR Am J Roentgenol 171: 107-113, 1998.

35. Lauffer RB, Parmelee DJ, Dunham SU, Ouellet HS, Dolan RP, Witte S, McMurry TJ and Walovitch RC: MS-325: albumintargeted contrast agent for MR angiography. Radiology 207: 529-538, 1998.

36. Cavagna FM, Lorusso V, Anelli PL, Maggioni F and de Haën C: Preclinical profile and clinical potential of gadocoletic acid trisodium salt (B22956/1), a new intravascular contrast medium for MRI. Acad Radiol 9 (Suppl 2): S491-S494, 2002.

37. Curtet C, Maton F, Havet T, Slinkin M, Mishra A, Chatal JF and Muller RN: Polylysine-Gd-DTPAn and polylysine-Gd-DOTAn coupled to anti-CEA $F\left(a b^{\prime}\right) 2$ fragments as potential immunocontrast agents. Relaxometry, biodistribution, and magnetic resonance imaging in nude mice grafted with human colorectal carcinoma. Invest Radiol 33: 752-761, 1998. 
38. Løkling KE, Fossheim SL, Skurtveit R, Bjørnerud A and Klaveness J: pH-sensitive paramagnetic liposomes as MRI contrast agents: in vitro feasibility studies. Magn Reson Imaging 19: 731-738, 2001.

39. Jung CW and Jacobs P: Physical and chemical properties of superparamagnetic iron oxide MR contrast agents: ferumoxides, ferumoxtran, ferumoxsil. Magn Reson Imaging 13: 661-674, 1995.

40. Taupitz M, Schnorr J, Abramjuk C, Wagner S, Pilgrimm H, Hünigen $\mathrm{H}$ and Hamm B: New generation of monomer-stabilized very small superparamagnetic iron oxide particles (VSOP) as contrast medium for MR angiography: preclinical results in rats and rabbits. J Magn Reson Imaging 12: 905-911, 2000.

41. Jung KH, Kim HK, Park JA, Nam KS, Lee GH, Chang Y and Kim TJ: Gd Complexes of DO3A-(Biphenyl-2,2'-bisamides) Conjugates as MRI Blood-Pool Contrast Agents. ACS Med Chem Lett 3: 1003-1007, 2012.

42. Bourrinet P, Bengele HH, Bonnemain B, Dencausse A, Idee JM Jacobs PM and Lewis JM: Preclinical safety and pharmacokinetic profile of ferumoxtran-10, an ultrasmall superparamagnetic iron oxide magnetic resonance contrast agent. Invest Radiol 41 $313-324,2006$
43. Weinmann HJ, Ebert W, Misselwitz B and Schmitt-Willich H: Tissue-specific MR contrast agents. Eur J Radiol 46: 33-44, 2003.

44. Corot C and Warlin D: Superparamagnetic iron oxide nanoparticles for MRI: contrast media pharmaceutical company R\&D perspective. Wiley Interdiscip Rev Nanomed Nanobiotechnol 5: 411-422, 2013.

45. Runge VM: A comparison of two MR hepatobiliary gadolinium chelates: Gd-BOPTA and Gd-EOB-DTPA. J Comput Assist Tomogr 22: 643-650, 1998.

46. Torres CG, Lundby B, Sterud AT, McGill S, Gordon PB and Bjerknes HS: MnDPDP for MR imaging of the liver - Results from the European phase III studies. Acta Radiol 38: 631-637, 1997. 